\title{
Study of the Competing Influence of Intermolecular Interactions of Different Types on the Structure of the Ferroelectrics
}

\author{
O.G. Maksimova ${ }^{a, *}$, V.I. Egorov ${ }^{a}$, R.A. Gerasimov ${ }^{b}$, A.R. Baidganov ${ }^{a}$ \\ AND A.V. MAKSIMOV ${ }^{a}$ \\ ${ }^{a}$ Cherepovets State University, Department of Physics, 162600 Cherepovets, Russian Federation \\ ${ }^{b}$ Southern Federal University, Department of Theoretical and Computer Hydro and Aerodynamics, \\ 344090, Rostov-on-Don, Russian Federation
}

\begin{abstract}
The modified Potts model considering the lattice size and the sublattice displacement is used for studying of structural transitions in ferroelectric systems. It was shown that the lattice type is determined by the competing influence of intermolecular interactions (dipolar-dipolar, exchange interactions, Lennard-Jones potential). The lattice parameters are calculated for different intermolecular interactions. It was shown that crystallographic parameters of lattice and the value of spontaneous polarization are changed drastically at a certain temperature.
\end{abstract}

DOI: 10.12693/APhysPolA.135.1084

PACS/topics: ferroelectric, orientational interactions, phase transitions, type of crystal lattice, Monte-Carlo method

\section{Introduction}

The main problem of statistical theories of ferroelectrics is that equilibrium properties are simulated for a predetermined crystal lattice. However, the lattice type and the crystallographic parameters could be changed under the influence of temperature, pressure and external electric fields. The Ising model is usually applied for simulations of the ferroelectric systems [1], that is, on the $180^{\circ}$-th domain wall model, which is a simplification, because there are configurations of the domain structure in which the direction of the polarization vector occurs at an angle different from $180^{\circ}$. Such a simplified approach is possible only to study the properties of substances with isolated axes outside the crystalline phase, for example, for the Rochelle salt or $\mathrm{KH}_{2} \mathrm{PO}_{4}$. In addition, the Ising model has a discrete symmetry group. Onsager had strictly proved that in this model there was a spontaneous polarization in a monolayer [2]. However, it was experimentally established that there are sufficiently thin ferroelectric films in which spontaneous polarization may be absent [3]. Therefore, the Potts model is used in this work.

\section{Model}

In low-molecular ferroelectric crystals, the polarization arises from the displacement of atoms of one sublattice to centers of faces (Fig. 1a) or nodes (Fig. 1b) of another sublattice. In this work, we substitute two sublattices for a three-dimensional lattice model of rotators for studying

*corresponding author; e-mail: og62@mail.ru

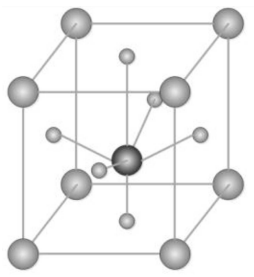

(a)

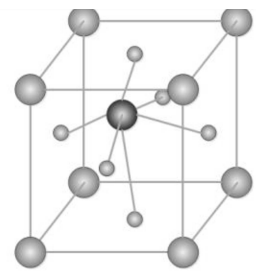

(b)

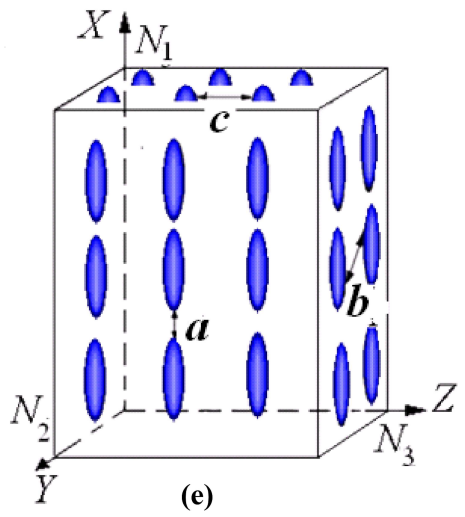

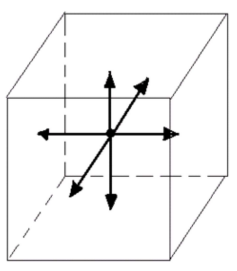

(d)

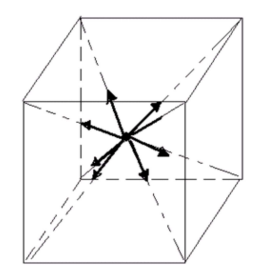

(e)
Fig. 1. (a) and (b) atomic displacement of the one sublattice of the ferroelectric crystal; (c) three-dimensional lattice model of rotators; (d) and (e) possible directions of rotators to the centers of faces (a), (d) and the nodes (b), (e) of another sublattice. 
of order and phase transitions in this system (Fig. 1c). The location of the node is determined by the set of three numbers $n_{1}, n_{2}, n_{3}$. A rotator (an element that has only rotational degrees of freedom) is related to each node. Possible directions of rotators, which depend on the types of sublattice displacement, are shown in Fig 1d and Fig 1e. Six directions of rotators are possible with the displacement of atoms to the centers of sublattice faces, eight directions are related to the displacement to the nodes.

In this work, the potential of the system consists of three terms: the Lennard-Jones potential, the potential of dipolar-dipolar interactions (the Keesom energy) and the potential of exchange interactions which is determined by the formula

$$
\begin{gathered}
U_{e x}=-\sum_{n_{1}, n_{2}, n_{3}} K_{1} \cos \left(\Phi_{n_{1}, n_{2}, n_{3}, n_{1}+1, n_{2}, n_{3}}\right) \\
-\sum_{n_{1}, n_{2}, n_{3}} K_{2} \cos \left(\Phi_{n_{1}, n_{2}, n_{3}, n_{1}, n_{2}+1, n_{3}}\right) \\
-\sum_{n_{1}, n_{2}, n_{3}} K_{3} \cos \left(\Phi_{n_{1}, n_{2}, n_{3}, n_{1}, n_{2}, n_{3}+1}\right) .
\end{gathered}
$$

Value of constants $K_{i}$ in formula (1) depends on the distance between rotators $r_{1}=a, r_{2}=b, r_{3}=c$, where $a, b, c$ are distances between dipoles in the directions $N_{1}$, $N_{2}, N_{3}$ respectively. In paper [1] it was shown, that for the dependence approximated by $K_{i}=K_{0} / r^{v}$, the value of $v$ is greater than 3. In this work $v$ is taken as 4 .

\section{Results and discussion}

At low temperatures, the rotators have a location in which the potential energy has a minimum. The dependencies of crystallographic distances at low temperatures are shown in Fig. 2 for different values of constants of exchange interactions $K_{0}$ on the relation $\lambda=k p^{2} / \varepsilon$, where $k$ is the constant in the Coulomb law, $p$ is a module of the dipole moment of rotators, $\varepsilon$ is the depth of the potential well at the Lennard-Jones potential. It was assumed that the crystallographic distances $b$ and $c$ are equal. One can see in Fig. 2 that the crystal lattice is cubic if the dipole-dipole interactions are neglected. As the value $\lambda$ increases, the parameter $b$ goes up whereas the value of $a$ becomes less, i.e., the anisotropy of the lattice increases. With further increase of the value $\lambda$, the parameters $a$ and $b$ appear to be equal to each other, and the lattice becomes cubic one.

For studying of the behavior of ferroelectric systems at high temperatures, the modified Potts model is used in which the sizes of crystal lattices and the selected directions are determined on each step of Monte-Carlo simulation and depend on the mutual position of sublattices (Fig. 1). The equilibrium state of the system is determined by the condition of minima of free energy. The dependencies of lattice parameters $a$ and $b$ on temperature are shown in Fig. 3a. The displacement of sublattice

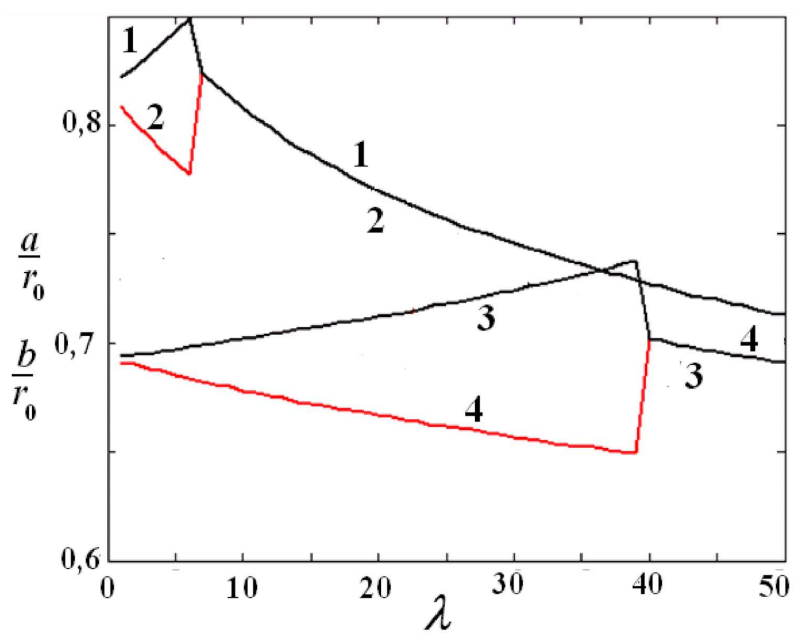

Fig. 2. Crystallographic lattice parameters $a$ (curves $2,4)$ and $b$ (curves 1,3 ) vs. the value $\lambda$ in low temperature for different constants of exchange interactions $K_{0}=10 \varepsilon$ (curves 1,2 ), $50 \varepsilon$ (curves 3,4 ). $r_{0}$ is the distance between rotators in the absence of orientational interactions.
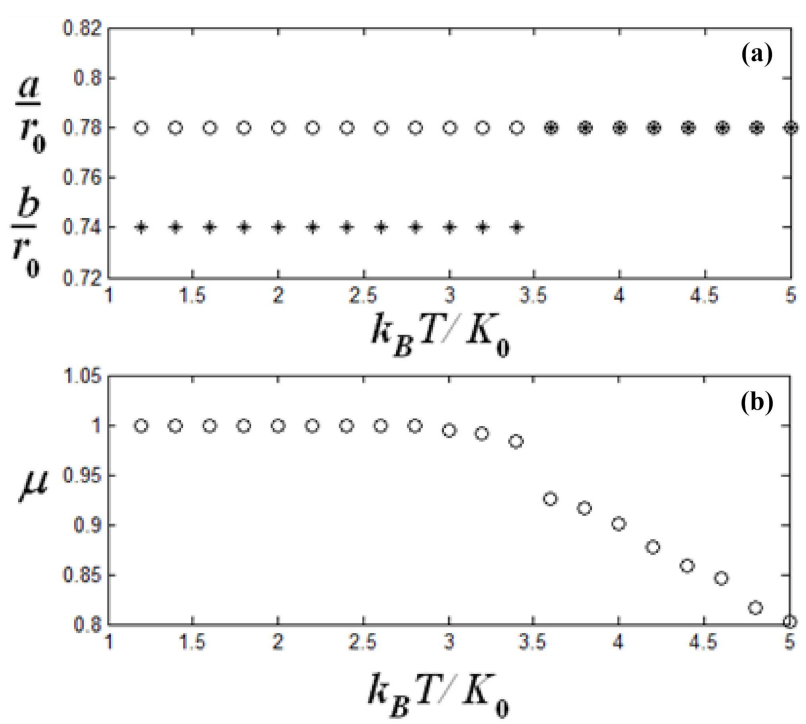

Fig. 3. (a) the crystallographic parameters: $a$ - upper row and $b$ - lower row, and (b) the dipole order vs. the normalized temperature.

and the decreasing of the dipole order parameter occur at a certain temperature (Fig. $3 \mathrm{~b}$ ), i.e. there is a phase transition at this point.

\section{Conclusion}

The influence of energy of orientational interactions becomes less significant with the increase of temperature. This fact influences the density of segments (rotators) and, accordingly, relative sizes of the system. 


\section{Acknowledgments}

The work is performed within the framework of the project "Methods of microstructural nonlinear analysis, wave dynamics and mechanics of composites for research and design of modern metamaterials and elements of structures made on its base" (grant No. 15-19-10008-P of the Russian Science Foundation).

\section{References}

[1] A.L. Pirozerskii, E.V. Charnaya, Phys. Solid State 52, 620 (2010).

[2] L. Onsager, Phys. Rev. 65, 117 (1944).

[3] V.M. Fridkin, Phys. Usp. 49, 193 (2006) (in Russian). 